\title{
Two Power Curves Yielding the Energy of a Lifetime in Evo-SETI Theory
}

\author{
Claudio Maccone \\ International Academy of Astronautics (IAA, Paris) \\ and Istituto Nazionale di Astrofisica (INAF, Italy)
}

Correspondence | claudio.maccone@gmail.com

Citation | Maccone, Claudio. 2020. "Two Power Curves Yielding the Energy of a Lifetime in Evo-SETI

Theory." Journal of Big History IV (2): xx-xx.

DOI | https://doi.org/10.22339/jbh.v4i2.4250

\begin{abstract}
This paper presents a new mathematical model for the ENERGY that a living being needs in order to live its whole life between birth and death. This also applies to a civilization made up by many living beings. The model is based on a LOGELL POWER CURVE, which is a curve in time made up by a lognormal probability density between birth and peak, followed by an ellipse between peak and death (LOGELL means LOGnormal plus ELLipse). We derive analytic equations yielding the ENERGY in terms of three free parameters only: the time of birth $b$, the time of the power peak $p$, and the time of death, $d$.

The author's previously published papers about his so-called Evo-SETI Theory (Evo-SETI stands for Evolution and SETI) cover the biological evolution over the last 3.5 billion years described as an increase in the number of living species from one (RNA) to the current (say) 50 million. Past mass extinctions make this evolution become a stochastic process having an exponential mean value, called Geometric Brownian Motion (GBM). In those papers, a lifetime, rather than a logell, was a b-lognormal, i.e., a lognormal starting at instant $b$ (birth) and descending straight to death at its descending inflexion point. Our mathematical discovery of the Peak-Locus Theorem showed that the GBM exponential is the geometric locus of all the peaks of the $b$-lognormals. Since $b$-lognormals are probability densities, the area under each of them always equals 1 (normalization condition); going from left to right on the time axis, the $b$-lognormals become more and more "peaky," so they last less and less in time. This "level of civilization" is what physicists call (Shannon) ENTROPY of information, meaning that the higher Species have higher information content than the lower Species. This author also proved mathematically that for all GBMs, the (Shannon) Entropy of the $b$-lognormals grows LINEARLY in time. The Molecular Clock, well known to geneticists since 1962, shows that the DNA base-substitutions occur LINEARLY in time since they are neutral with respect to Darwinian selection. The conclusion is that the Molecular Clock and the LINEAR increase of EvoEntropy in time are just the same thing! In other words, we derived the Molecular Clock mathematically as a part of our Evo-SETI Theory. Finally, this linearly growing entropy is just the new EvoSETI SCALE to measure the evolution of life on Exoplanets (measured in bits). In conclusion, our invention of the logell power curve, described in this paper, provides a new mathematical tool for our Evo-SETI mathematical description of Life, History and SETI.
\end{abstract}

Keywords: Biological Evolution; Molecular Clock; Entropy; SETI. 


\section{PART 1: Logell Curves and Their History Equations}

\section{Introduction to Logell "Finite Lifetime" Curves}

The starting idea is easy: we seek to represent the lifetime of any living being by virtue of just three points in the time: birth, peak, death (BPD). No other point in between them is needed. That is, no other "senility point" $s$ is needed such as the one appearing in all b-lognormals that this author had published in his Evo-SETI Theory prior to 2017. In fact, it is easier and more natural to describe someone's lifetime in terms of just birth, peak and death, than in terms of birth, senility and death, since it is uncertain to define when senility arrives, and actually difficult to define in the practice for any individual or for any civilization.

Please look at Figure 1. The first part, the one on the left, i.e., prior to the peak time $p$, is just a blognormal: it starts at birth time $b$, climbs up to the adolescence time $a$ (ascending inflexion point of the b-lognormal) (in reality the adolescence time should more properly be called "puberty time" since it marks the beginning of the reproduction capacity for that individual) and finally reaches the peak time at $p$ (maximum, i.e., the point of zero first derivative of the b-lognormal). All this is just ordinary $\mathbf{b}$ lognormal stuff, as we have been publishing since about 2012.

Now the novelty comes, i.e., the second part, the one on the right: that is just a ellipse having its vertex exactly at the peak time $p$. Notice that this definition automatically implies that the tangent line at the peak is horizontal, i.e., the same for both the b-lognormal and the ellipse. Notice also that, after the peak, the ellipse plunges down until it reaches the time axis at the death time $d$ with a perfectly vertical tangent. Therefore, this new definition of death time $d$ is different from the old definition of $d$ applying to b-lognormals alone, as we published prior to 2017.

This is the LOGELL (b-LOGnormal plus ELLipse) new CURVE, FINITE IN TIME (namely ranging in time just between birth and death). We introduce the logell for the first time in the present paper and we study it with surprising results.

History of the ROMAN CIVILIZATION as a LOGELL finite curve

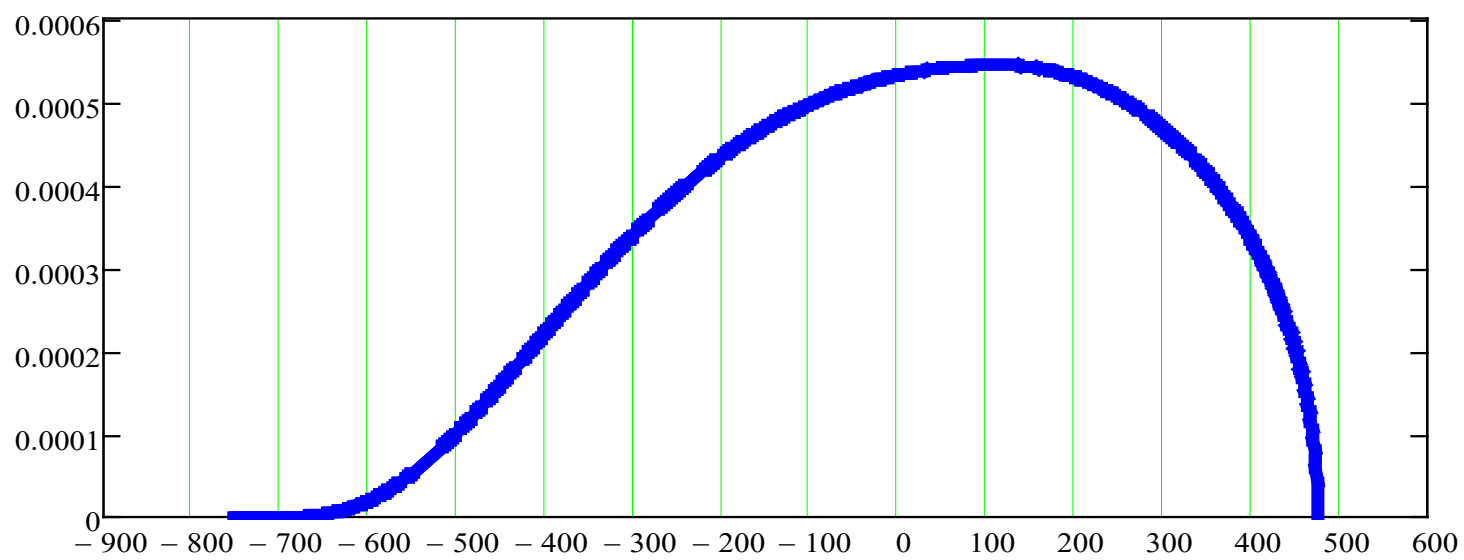

Figure 1. Representation of the History of the Ancient Roman civilization as a LOGELL curve, finite in the time. Rome was funded in 753 B.C., i.e., in the year -753 in our notation, or $b=-753$. Then the Roman republic and empire (the latter since the first emperor, Augustus, roughly after 27 B.C.) kept growing in conquered territory until it reached its peak (maximum extension, up to Susa in current Iran) in the year 117 A.D., i.e., $p=117$, under emperor Trajan. Afterward, it started to decline and loose territory until the final collapse in 476 A.D. ( $d=476$, Romulus Augustulus, last emperor). Thus, just three points in time are necessary to summarize the History of Rome: $b=-753, p=117, d=476$. The numbers along the vertical axis will be explained later. 
History of the ROMAN CIVILIZATION as a LOGPAR finite curve

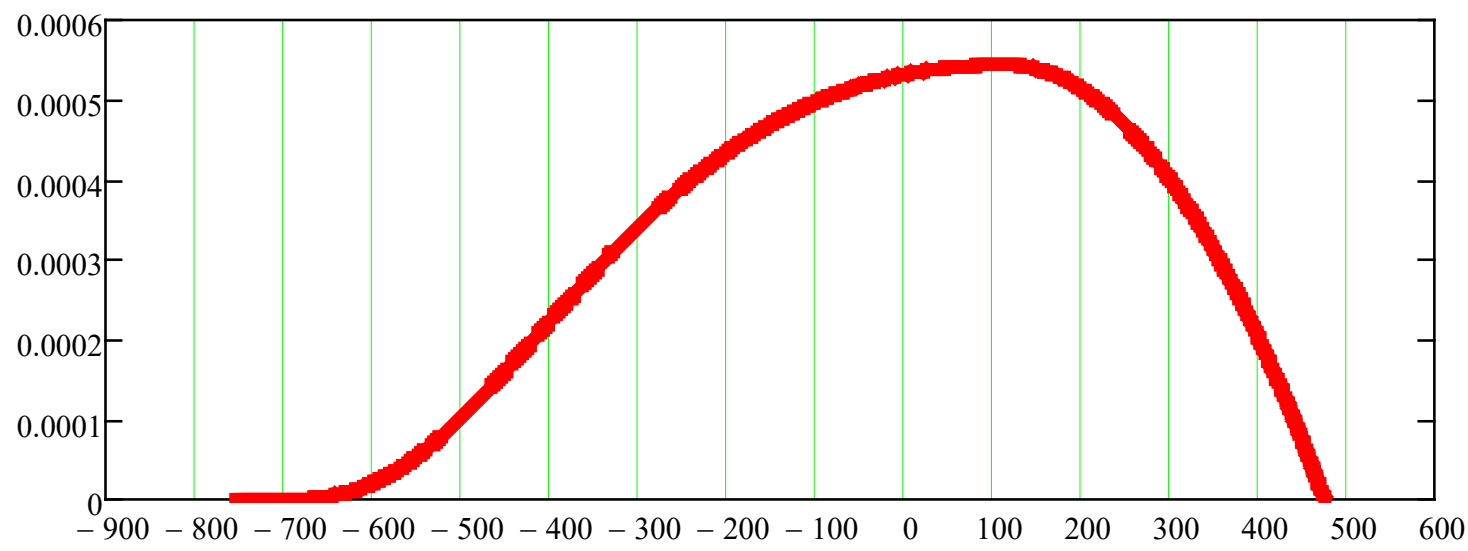

Figure 2. Representation of the History of the Roman civilization as a LOGPAR finite curve. This logpar curve in time is made up by a b-lognormal in between birth and peak, and a parabola in between peak and death. It was used by this author in the years 2017 and 2018 in refs. [1] and [2]. Please have a look at these references for the full logpar mathematical description, culminating in the two Logpar History Formulae expressing the b-lognormal's two parameters $\mu$ (a real number) and $\sigma$ (a positive number) in terms of the three assigned real numbers $b, p$ and $d$, with the condition $b<p<d$.

Rome's LOGPAR (red) and LOGELL (red before peak, blue after peak)

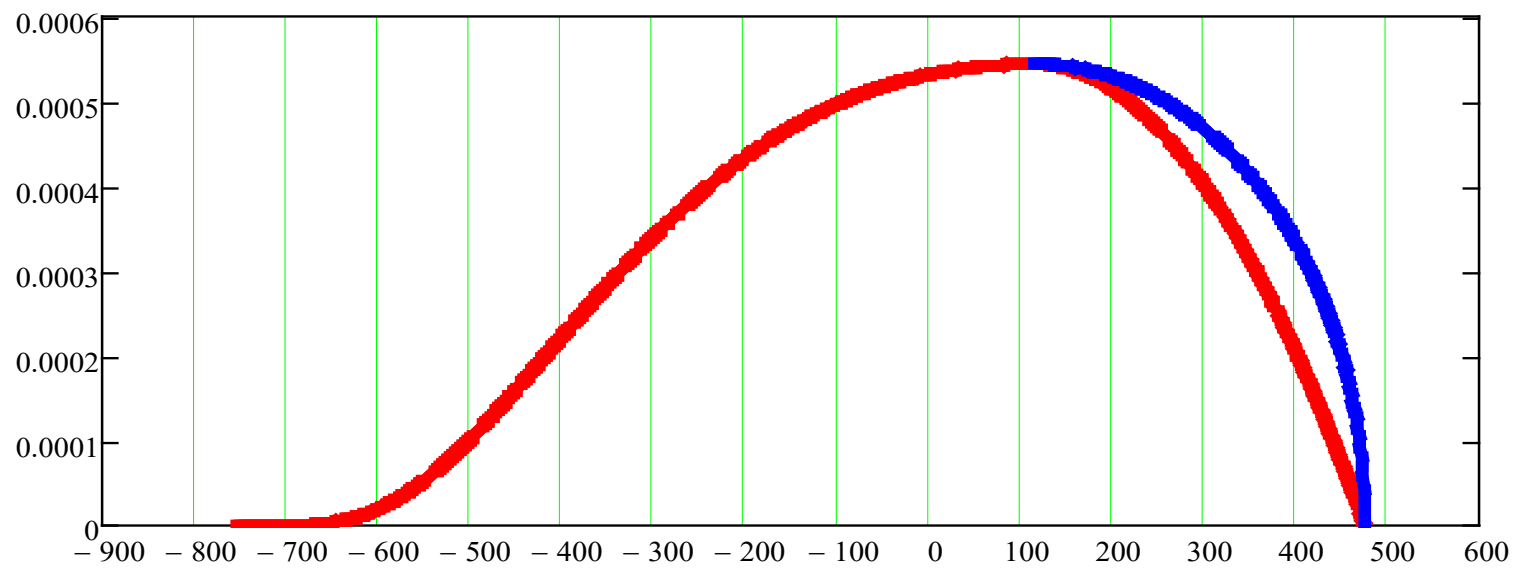

Figure 3. The difference between LOGELL and LOGPAR is only in their behaviour between peak $p$ and death $d$ : an ELLIPSE for the LOGELL (in blue above) and a PARABOLA for the LOGPAR (in red above). The common part of the curve prior to the peak (shown in red here) is a b-lognormal, that is a lognormal probability density function (pdf) in the time starting at the birth time $b$ and reaching its peak at time $p$. In this way, the finite lifetime of any living being or civilization is a POWER curve (power means measured in Watts, as in physics) and the area under this power curve is the total ENERGY that the living being or civilization needs in order to cope for its own existence. In fact, the ENERGY is the just integral of the POWER in the time, or, if you prefer, the POWER is just the DERIVATIVE OF THE ENERGY with respect to the time. 


\section{Finding the Ellipse Equation of the Right Part of the Logell}

We will now cast into appropriate mathematics the above popular description of a logell curve. Consider the equation of an ellipse in the time $t$ having vertical axis along the $t=p$ vertical line:

$$
\frac{x^{2}}{a^{2}}+\frac{y^{2}}{b^{2}}=1
$$

as every high-school student knows. The horizontal axis now is the time, denoted $t$, with $p<t<d$. On the vertical axis, we have the independent variable $y$ ranging between o and $P$. Thus, we may pass from (1) to the new ellipse equation by virtue of the four substitutions

$$
\left\{\begin{array}{c}
x \rightarrow(t-p) \\
a \rightarrow(d-p) \\
y \rightarrow y \\
b \rightarrow P
\end{array}\right.
$$

Inserting (2) into (1), the latter becomes

$$
\frac{(t-p)^{2}}{(d-p)^{2}}+\frac{y^{2}}{P^{2}}=1
$$

That is

$$
\frac{y^{2}}{P^{2}}=1-\frac{(t-p)^{2}}{(d-p)^{2}}
$$

Solving (4) for $y$ and taking the plus sign in front of the radical ,i.e., the upper branch of the ellipse, yields the desired equation of our quarter of the ellipse as a function of the time $t$

$$
y(t)=P \sqrt{1-\frac{(t-p)^{2}}{(d-p)^{2}}} .
$$

As confirmation, one may check that (5) immediately yields the two evident conditions

$$
\left\{\begin{array}{l}
y(p)=P \\
y(d)=0 .
\end{array}\right.
$$

3. Finding the b-Lognormal Equation of the Left Park of the Logell

As for the b-lognormal between birth and peak, the left part of the logell curve, we already know all its mathematical details from the previous many papers published by this author on this topic, but we shall summarize here the main equations for the sake of completeness.

The equation of the b-lognormal starting at $b$ reads

$$
\text { b_lognormal }(t ; \mu, \sigma, b)=\frac{e^{-\frac{(\log (t-b)-\mu)^{2}}{2 \sigma^{2}}}}{\sqrt{2 \pi} \sigma(t-b)} \text {. }
$$

with $\sigma>0$ and $-\infty<\mu<\infty$. Tables listing the main equations that can be derived from (7) were given by this author in refs. [1] and [2] and we shall not re-derive them here again. We just confine ourselves to reminding that: 
1) The abscissa $p$ of the peak of (7) is given by

$$
p=b+e^{\mu-\sigma^{2}} .
$$

Proof. Take the derivative of (7) with respect to $t$ and set it equal to zero. Then solve the resulting equation for $t$, which now becomes $p$, and (8) is found.

2) The ordinate $P$ of the peak of (7) is given by

$$
P=\frac{e^{\frac{\sigma^{2}}{2}-\mu}}{\sqrt{2 \pi} \sigma} .
$$

Proof. Rewrite $p$ instead of $t$ in (7) and then insert (8) instead of $p$. Then simplify to find (9).

3) The abscissa of the adolescence point (that should actually be better named "puberty point") is the abscissa of the ascending inflexion point of $(7)$. It is given by

$$
a=b+e^{-\frac{\sigma \sqrt{\sigma^{2}+4}}{2}-\frac{3 \sigma^{2}}{2}+\mu}
$$

Proof. Take the second derivative of (7) with respect to $t$ and set it equal to zero. Then solve the resulting equation for $t$, that now becomes $a$, and (10) is found.

4) The ordinate of the adolescence point is given by

$$
\frac{e^{-\frac{\sigma \sqrt{\sigma^{2}+4}}{4}+\frac{\sigma^{2}}{4}-\mu-\frac{1}{2}}}{\sqrt{2 \pi} \sigma}
$$

Proof. Just rewrite $a$ instead of $t$ in (7) and then insert (10) and simplify the result.

Let us now notice that, within the framework of the logell theory described in this paper, we may NOT say that (7) fulfills the b-lognormal's normalization condition

$$
\int_{b}^{\infty} \mathrm{b} \_\operatorname{lognormal}(t ; \mu, \sigma, b) d t=1
$$

In fact, (7) here is allowed to range in between only $b$ and $p$. Thus, rather than adopting (12), we must thus replace (12) by the integral of (7) between $b$ and $p$ only. Fortunately, it is possible to evaluate this integral in terms of the error function defined by

$$
\operatorname{erf}(x)=\frac{2}{\sqrt{\pi}} \int_{0}^{x} e^{-t^{2}} d t
$$

In fact, the integral of the b-lognormal (7) between $b$ and $p$ turns out to be given by

$$
\begin{aligned}
& \int_{b}^{p} \mathrm{~b}_{-} \operatorname{lognormal}(t ; \mu, \sigma, b) d t= \\
& \quad=\int_{b}^{p} \frac{e^{-\frac{(\log (t-b)-\mu)^{2}}{2 \sigma^{2}}}}{\sqrt{2 \pi} \sigma(t-b)} d t=\frac{1+\operatorname{erf}\left(\frac{\sqrt{2} \ln (p-b)-\sqrt{2} \mu}{2 \sigma}\right)}{2}=
\end{aligned}
$$


Now, inserting (8) instead of $p$ into the last erf argument, a remarkable simplification occurs: $\mu$ and $b$ both disappear and only $\sigma$ is left. In addition, the erf property $\operatorname{erf}(-x)=-\operatorname{erf}(x)$ allows us to rewrite

$$
=\frac{1+\operatorname{erf}\left(-\frac{\sigma}{\sqrt{2}}\right)}{2}=\frac{1-\operatorname{erf}\left(\frac{\sigma}{\sqrt{2}}\right)}{2} .
$$

In conclusion, the area under the b-lognormal between birth and peak is given by

$$
\int_{b}^{p} \mathrm{~b} \_\operatorname{lognormal}(t ; \mu, \sigma, b) d t=\frac{1-\operatorname{erf}\left(\frac{\sigma}{\sqrt{2}}\right)}{2} .
$$

This result will prove to be of key importance for the further developments described in the present paper.

\section{Area under the Ellipse on the Right Part of the Logell between Peak and Death}

We already proved that the ellipse on the right part of the logell curve has the equation (5). Now we want to find the area under this ellipse between peak and death. If one remembers that the area of the whole ellipse with semi-axes $a$ and $b$ equals $\pi a b$, it is obvious that our area is just a quarter of that. The same result is, of course, found by evaluating the definite integral (this calculation left to the reader as an exercise)

$$
\int_{p}^{d} P \sqrt{1-\frac{(t-p)^{2}}{(d-p)^{2}}} d t=\frac{\pi}{4} P(d-p) .
$$

\section{Area under the Full Logell Curve between Birth and Death}

We are now in a position to compute the full area $A$ under the logell curve, that is given by the sum of equations (15) and (16), that is

$$
\frac{1-\operatorname{erf}\left(\frac{\sigma}{\sqrt{2}}\right)}{2}+\frac{\pi}{4} P(d-p)=A
$$

This is one of the most important equations in this paper. In fact, if we want the logell be a truly probability density function (pdf), we must assume in (17)

$$
A=1 .
$$

But, surprisingly, we shall NOT do so! Let us rather ponder over what we are doing:

1) We are creating a "Mathematical History" model where the "unfolding History" of each Civilization in the time is represented by a logell curve.

2) The knowledge of only three points in time is requested in this model: $b, p$ and $d$.

3) But the area under the whole curve depends on $\sigma$ as well as on $\mu$, as we see upon inserting (9) instead of $P$ into (17), that is

$$
\frac{1-\operatorname{erf}\left(\frac{\sigma}{\sqrt{2}}\right)}{2}+\frac{e^{\frac{\sigma^{2}}{2}-\mu}}{\sqrt{2 \pi} \sigma} \cdot \frac{\pi}{4}(d-p)=A(\mu, \sigma) .
$$


4) Also, $p$ is to be replaced by its expression (8) in terms of $\sigma$ and $\mu$, yielding the new equation

$$
\frac{1-\operatorname{erf}\left(\frac{\sigma}{\sqrt{2}}\right)}{2}+\frac{e^{\frac{\sigma^{2}}{2}-\mu(\sigma)}}{\sqrt{2 \pi} \sigma} \cdot \frac{\pi\left(d-b-e^{\mu(\sigma)-\sigma^{2}}\right)}{4}=A(\sigma) \cdot \text { (20) }
$$

5) The meaning of (20) is that if birth and death are fixed, but the position of the peak may freely move in between them according to the different living beings or civilizations that we are going to consider. In other words, (20) yields different numeric values of $\sigma$ and $\mu(\sigma)$ according to where the peak is between birth and death.

6) In addition, we would like to get rid of the error function erf in (20). How may we do so?

6. The Area under the Logell Curve Depends on Sigma Only, and Here is the Area Derivative W.R.T. Sigma

The simple answer to the last question 6) is "by differentiating both sides of (20) with respect to $\sigma$." In fact, the derivative of the erf function (13) is just the "Gaussian" exponential,

$$
\frac{d \operatorname{erf}(x)}{d x}=\frac{2}{\sqrt{\pi}} \cdot e^{-x^{2}} .
$$

Therefore, the erf function itself will disappear by differentiating (20) with respect to $\sigma$. Actually, the derivative of the first term on the left side of (20) simply is, according to (21),

$$
\frac{d}{d \sigma}\left[\frac{1-\operatorname{erf}\left(\frac{\sigma}{\sqrt{2}}\right)}{2}\right]=-\frac{e^{-\frac{\sigma^{2}}{2}}}{\sqrt{2 \pi}} .
$$

As for the derivative with respect to $\sigma$ of the second term on the left side of (20), we firstly notice that $\sigma$ appears three times within that term. Thus, the relevant derivative is the sum of three terms, each of which includes the derivative of one of the three terms multiplied by the other two terms unchanged. In equations, one has the following:

$$
\begin{aligned}
& \frac{d}{d \sigma}\left[\frac{1-e r f\left(\frac{\sigma}{\sqrt{2}}\right)}{2}+\frac{e^{\frac{\sigma^{2}}{2}-\mu}}{\sqrt{2 \pi} \sigma} \cdot \frac{\pi\left(d-b-e^{\mu-\sigma^{2}}\right)}{4}\right]= \\
& =\frac{\sqrt{\pi} e^{-\frac{\sigma^{2}}{2}}}{2^{\frac{3}{2}}}-\frac{e^{-\frac{\sigma^{2}}{2}}}{\sqrt{2 \pi}}-\frac{\sqrt{\pi}\left(-e^{\mu-\sigma^{2}}+d-b\right) e^{\frac{\sigma^{2}}{2}-\mu}}{2^{\frac{5}{2}} \sigma^{2}}+ \\
& -\frac{\sqrt{\pi}\left(e^{\mu-\sigma^{2}}-d+b\right) e^{\frac{\sigma^{2}}{2}-\mu}}{2^{\frac{5}{2}}} .
\end{aligned}
$$

Several alternative forms of this equation (23) are possible, and that is rather confusing. However, using a symbolic manipulator (this author did do so by virtue of NASA's Maxima), a few more steps lead to the following form of (23): 


$$
\begin{aligned}
& \frac{d A(\mu(\sigma), \sigma)}{d \sigma} \equiv \frac{d A(\sigma)}{d \sigma}= \\
& =\frac{e^{-\frac{\sigma^{2}}{2}+\mu} \sqrt{2}\left[\pi(d-b)\left(\sigma^{2}-1\right) e^{\sigma^{2}}+e^{\mu}\left(\sigma^{2}(\pi-4)+\pi\right)\right]}{8 \sqrt{\pi} \sigma^{2}} .
\end{aligned}
$$

We may further simplify (24) by inserting (8) and so letting $\mu$ disappear. The result is

$$
\begin{aligned}
& \frac{d A(\sigma)}{d \sigma}= \\
& ==\frac{e^{-\frac{\sigma^{2}}{2}+\mu} \sqrt{2}\left\{\pi(d-p)\left(\sigma^{2}-1\right) e^{\sigma^{2}}+e^{\sigma^{2}}(p-b)\left[\sigma^{2}(\pi-4)+\pi\right]\right\}}{8 \sqrt{\pi} \sigma^{2}}= \\
& =\frac{e^{-\frac{\sigma^{2}}{2}+\mu} \sqrt{2} e^{\sigma^{2}}\left\{\pi(d-p)\left(\sigma^{2}-1\right)+(p-b)\left[\sigma^{2}(\pi-4)+\pi\right]\right\}}{8 \sqrt{\pi} \sigma^{2}}= \\
& =\frac{e^{\frac{\sigma^{2}}{2}+\mu} \sqrt{2}\left\{\sigma^{2}[\pi(d-b)+(\pi-4)(p-b)]-(\pi(d-p))\right\}}{8 \sqrt{\pi} \sigma^{2}} .
\end{aligned}
$$

This (25) is the derivative of the logell area with respect to sigma.

\section{Exact "History Equations" for each Logell Curve}

We now take a further, crucial step in our analysis of the logell curve: we IMPOSE that the derivative of the area with respect to sigma, i.e., (25), is zero

$$
\frac{d A(\sigma)}{d \sigma}=0
$$

What does that mean? Well, (26) is the Evo-SETI equivalent of the LEAST ACTION PRINCIPLE in physics! This conclusion does not show up at the moment, but it will at the end of this paper.

For the time being, rewrite the imposed condition (26) by virtue of the last expression in (25) that, getting rid of both the exponential and the denominator, boils down to

$$
\sigma^{2}[\pi(d-b)+(\pi-4)(p-b)]-(\pi(d-p))=0 . \quad(27)
$$

The last equation is a quadratic equation in $\sigma$

$$
\sigma^{2}[\pi(d-b)+(\pi-4)(p-b)]=\pi(d-p)
$$

that, solved for $\sigma^{2}$, immediately yields

$$
\sigma^{2}=\frac{\pi(d-p)}{\pi(d-b)+(\pi-4)(p-b)} .
$$

This is the most important new result discovered in the present paper: the LOGELL HISTORY EQUATION FOR $\sigma$,

$$
\sigma=\frac{\sqrt{\pi} \sqrt{d-p}}{\sqrt{\pi(d-b)+(\pi-4)(p-b)}} .
$$


In words, given the input triplet $(b, p, d)$, then (3o) immediately yields the exact $\sigma$ of the elliptical left part of the logell curve. It was discovered by this author on September 4, 2018, and led not only to this paper but to the introduction of the ENERGY spent in a lifetime by a living creature, or by a whole civilization whose "power-vs-time" behaviour is given by the logell curve, as we will understand better in the coming sections of this paper. At the moment we confine ourselves to taking the limit of both sides of (30) for $d \rightarrow \infty$, with the result

$$
\begin{aligned}
\lim _{d \rightarrow \infty} \sigma & =\lim _{d \rightarrow \infty} \frac{\sqrt{\pi} \sqrt{d-p}}{\sqrt{\pi(d-b)+(\pi-4)(p-b)}}= \\
& =\lim _{d \rightarrow \infty} \frac{\sqrt{\pi} \sqrt{d}}{\sqrt{\pi d}}=1 .
\end{aligned}
$$

Since we already know that $\sigma$ must be positive (31) really shows that $\sigma$ may range between zero and one only

$$
0<\sigma<1 .
$$

The two limiting values $\sigma \rightarrow 0$ and $\sigma \rightarrow 1$ are "unphysical", since one may not die at birth nor die at an infinite age, respectively.

Next to (30), of course, is a similar LOGELL EQUATION FOR $\mu$, which is immediately derived from (8) and (30). To this end, just take the log of (8) to get

$$
\mu=\ln (p-b)+\sigma^{2}
$$

so that, invoking (29), one finds the desired logell equation for $\mu$ :

$$
\mu=\ln (p-b)+\frac{\pi(d-p)}{\pi(d-b)+(\pi-4)(p-b)}
$$

In conclusion, our key two LOGELL HISTORY EQUATIONS are

$$
\left\{\begin{array}{l}
\sigma=\frac{\sqrt{\pi} \sqrt{d-b}}{\sqrt{\pi(d-b)+(\pi-4)(p-b)}} \\
\mu=\ln (p-b)+\frac{\pi(d-p)}{\pi(d-b)+(\pi-4)(p-b)} .
\end{array}\right.
$$

\section{Considerations on the Logell History Equations}

Some considerations on the logell History Formulae (35) are now in order:

1) All these formulae are exact, i.e., no Taylor series expansion was used to derive them.

2) But they were obtained by equalling to zero the derivative with respect to $\sigma$ of the total area under the logell curve given by (20).

3) Therefore, the logell History Formulae (35) are the equations of a minimum (later shown that this is, indeed, a minimum and not a maximum) of the $A(\sigma)$ function expressing the total area (20) as a function of $\sigma$. This minimum is the MINIMUM ENERGY PRINCIPLE of our Evo-SETI Theory, i.e., "in our lifetime, we always act in such a way as to minimize the energy that we are using."

9. Logell Peak Coordinates Expressed in Terms of $(b, p, d)$ Only 
Of particular importance for all future logell applications is the expression of the peak coordinates $(p, P)$ expressed in terms of the input triplet $(b, p, d)$ only. Since the peak abscissa $p$ is assumed to be known, we have to derive only the formula for the peak ordinate $P$. That is readily obtained by inserting the logell History Formulae (35) into the peak height expression (9). After a few rearrangements, it is found to be given by

$$
P=\frac{\sqrt{(\pi-4)(p-b)+\pi(d-b)}}{\sqrt{2} \pi \sqrt{d-p}(p-b)} e^{-\frac{\pi(d-p)}{2[(\pi-4)(p-b)+\pi(d-b)]}} \cdot \text { (36) }
$$

10. History of Rome as an Example of How to Use the Logell History Formulae

Let us go back to the History of Rome as summarized in the caption to Figure 1. First of all, let us write down neatly the key three numeric input values in the History of Rome that were already mentioned in the caption to Figure 1:

$$
\text { Rome_input_triplet }=\left\{\begin{array}{l}
b=-753 \\
p=117 \\
d=476 .
\end{array}\right.
$$

Then the logell History Equations (35) immediately yield numerical values of the logell $\sigma$ and $\mu$ for Rome, which we shall hereafter denote by $\sigma R$ and $\mu R$, respectively

$$
\text { Rome_logell_doublet }\left\{\begin{array}{l}
\sigma R=0.602 \\
\mu R=7.131 .
\end{array}\right.
$$

Next the study of the logell peak comes. We already know from (37) that the abscissa of the peak of the Roman civilization was in 117 A.D. under Trajan

$$
p_{\text {Rome }}=117 \text {. }
$$

However, the logell peak ordinate for Rome must be found by virtue of (36). One thus gets

$$
P_{\text {logell } \_ \text {Rome }}=6.358 \cdot 10^{-4}=0.0006358 . \quad(40)
$$

Part 2: Energy as the Area under Logell Power Curves

11. Area under Any Logell Power Curve and its Meaning as "Lifetime Energy" of That Living Being

The logell history formulae (35)

$$
\left\{\begin{array}{l}
\sigma=\frac{\sqrt{\pi} \sqrt{d-p}}{\sqrt{\pi(d-b)+(\pi-4)(p-b)}} \\
\mu=\ln (p-b)+\frac{\pi(d-p)}{\pi(d-b)+(\pi-4)(p-b)} .
\end{array}\right.
$$

are "much similar" to the logpar history formulae (39) of ref. [1], that is 


$$
\left\{\begin{array}{l}
\sigma=\frac{\sqrt{2} \sqrt{d-p}}{\sqrt{2 d-(b+p)}} \\
\mu=\ln (p-b)+\frac{2(d-p)}{2 d-(b+p)}
\end{array}\right.
$$

From this point in the paper onward, we may thus proceed along the lines outlined in ref. [1]. What is the physical meaning of the area (20)? If we consider the logell curve as the curve of the power (measured in watts) of the Roman civilization along the whole of its history course, then the area under this curve, i.e., the integral of the logell between birth and death is the total energy (measured in joules) spent by the civilization in its whole lifetime:

$$
\begin{aligned}
& \text { ENERGY_spent_in_the_Civilization_LIFETIME }= \\
& =\int_{\mathrm{b}}^{\mathrm{d}} \text { POWER_of_that_Civilization }(t) d t= \\
& =\int_{\mathrm{b}}^{\mathrm{d}} \text { logell_curve_of_that_Civilization }(t) d t .
\end{aligned}
$$

In other words, if we know the power curve of any living being that lived in the past, like a cell, or an animal, or a human, or a civilization of humans or of any other living forms (including extraterrestrials), the integral of that power curve, i.e., logell curve, between birth and death, is the TOTAL ENERGY spent by that living form during the whole of its lifetime.

One more point regarding the last statement is this: if we assume that all Humans have potentially the same amount of energy to spend during their whole lifetime, then the logell of great men who "died young" (like Mozart, for instance) must have the same area below their logell and so a much higher peak since they lived shorter than others.

Let us next consider the condition that usually one's death comes after one's peak, but, in exceptional cases, one's death may also come just at one's peak (as, for instance, for death in a car accident), that is

$$
d \geq p
$$

We now wish to find the value of the ENERGY AT THE PEAK of one's lifetime. Then, taking the limit of (19) for $d \rightarrow p$ from above and noticing from (13) that $\operatorname{erf}(0)=0$, we find

$$
\begin{aligned}
& \operatorname{Energy}(p)=\lim _{d \rightarrow p} \frac{1-\operatorname{erf}\left(\frac{\sigma}{\sqrt{2}}\right)}{2}= \\
& =\lim _{d \rightarrow p} \frac{1-\operatorname{erf}\left(\frac{\sqrt{\pi} \sqrt{d-p}}{\sqrt{2} \sqrt{\pi(d-b)+(\pi-4)(p-b)}}\right)}{2}= \\
& =\frac{1-\operatorname{erf}(0)}{2}=\frac{1}{2} .
\end{aligned}
$$

This (45) may look like a surprising result: why should the Energy of a being at its peak be equal to just $1 / 2$ and not to any other positive value (in joules)? Well, we will soon solve this matter of extending the Energy at peak from $1 / 2$ to any other positive value as we did already in ref. [1]. But, for the time being, please just content yourself with using the conventional value $1 / 2$ to simplify the calculations.

On the contrary, if we take the limit of (19) for $d \rightarrow \infty$ (which limit is called "immortality limit" since the living being or civilization is now supposed to live for an infinite amount of time), we immediately see that 


$$
\begin{aligned}
& \lim _{d \rightarrow \infty} A(\mu, \sigma)= \\
& =\lim _{d \rightarrow \infty}\left\{\frac{1-\operatorname{erf}\left(\frac{\sigma}{\sqrt{2}}\right)}{2}+\frac{e^{\frac{\sigma^{2}}{2}}-\mu}{\sqrt{2 \pi} \sigma} \cdot \frac{\pi}{4}(d-p)\right\}=\infty .
\end{aligned}
$$

In other words: "if you want to live for an infinite amount of time, you need an infinite amount of energy to do so" (as is obvious).

The next question is this: what is the time of the energy minimum? We skip all the lengthy calculations made by Maxima and just write here the result:

$$
\begin{aligned}
& d_{\text {abscissa_of_logel__minimum_Energy }}= \\
& =b+\frac{\left(\sqrt{-\pi^{2}+8 \pi-12}+2\right)(p-b)}{\pi} .
\end{aligned}
$$

This is the abscissa of the minimum of the Energy: we could prove that it is really a minimum, rather than a maximum, by computing the second derivative, but we shall not do so here for the sake of brevity. Let us rather remark that, for the case (37) of Ancient Rome, (47) yields the year of Rome's minimum energy as 301 A.D. This was the time of Emperor Diocletian, certainly a very troubled time since it was the time of the largest persecution ever against the Christians (https://en.wikipedia.org/wiki/Diocletian). Tourists, visiting the Basilica of Santa Maria degli Angeli e dei Martiri in today's Rome, might just think of the 40,0oo Christian slaves who died to build Diocletian's Baths, e.g., just at the minimum of Pagan Rome's Civilization.

\section{Discovering an Oblique Asymptote of the Energy Function Energy $(D)$ Is Increasing Indefinitely}

This author discovered (on September 4, 2018) that the following logell total energy (48) has an oblique asymptote for $d \rightarrow \infty$. Before we derive the equation of this oblique asymptote, however, some careful understanding of what $d$ means is in order. We always said that the logell theory described in this paper necessitates the three inputs $(b, p, d)$. However, in this section, we are going to consider higher

and higher values of the death instant $d$ so that the area under the logell, i.e., the energy of the phenomenon of which the logell is the power, may assume any assigned value. Thus, in this section, the death time $d$ becomes a sort of new independent variable $D$ rather than just one of the three fixed inputs $(b, p, d)$. In other words still, we will be careful to make the distinction between

1) the fixed, i.e., known, death instant $d$ and

2) the movable, i.e. independent variable $D$, allowing us to extrapolate into the future the logell

having the three fixed input values $(b, p, d)$.

Having so said, the logell total ENERGY provided by Maxima upon inserting (41) into (20) and then rearranging must more correctly be rewritten as a function of $D$ rather than $d$ and reads logell_total_energy $(D)=$

$$
\begin{aligned}
& =\frac{1-\operatorname{erf}\left(\frac{\sqrt{\pi} \sqrt{D-p}}{\sqrt{2} \sqrt{\pi(D-b)+(\pi-4)(p-b)}}\right)}{2}+ \\
& +\frac{\sqrt{D-p} \sqrt{\pi(D-b)+(\pi-4)(p-b)} e^{-\frac{\pi(D-p)}{2 \pi D+(2 \pi-8) p+(8-4 \pi) b}}}{2^{\frac{5}{2}}(p-b)} .
\end{aligned}
$$


The following Figure 4 shows Rome's both LOGELL total energy in blue and the LOGPAR total energy in red, the latter as mathematically described in ref. [1].

\section{Rome's LOGELL energy (blue) and LOGPAR energy (red) with asymptotes}

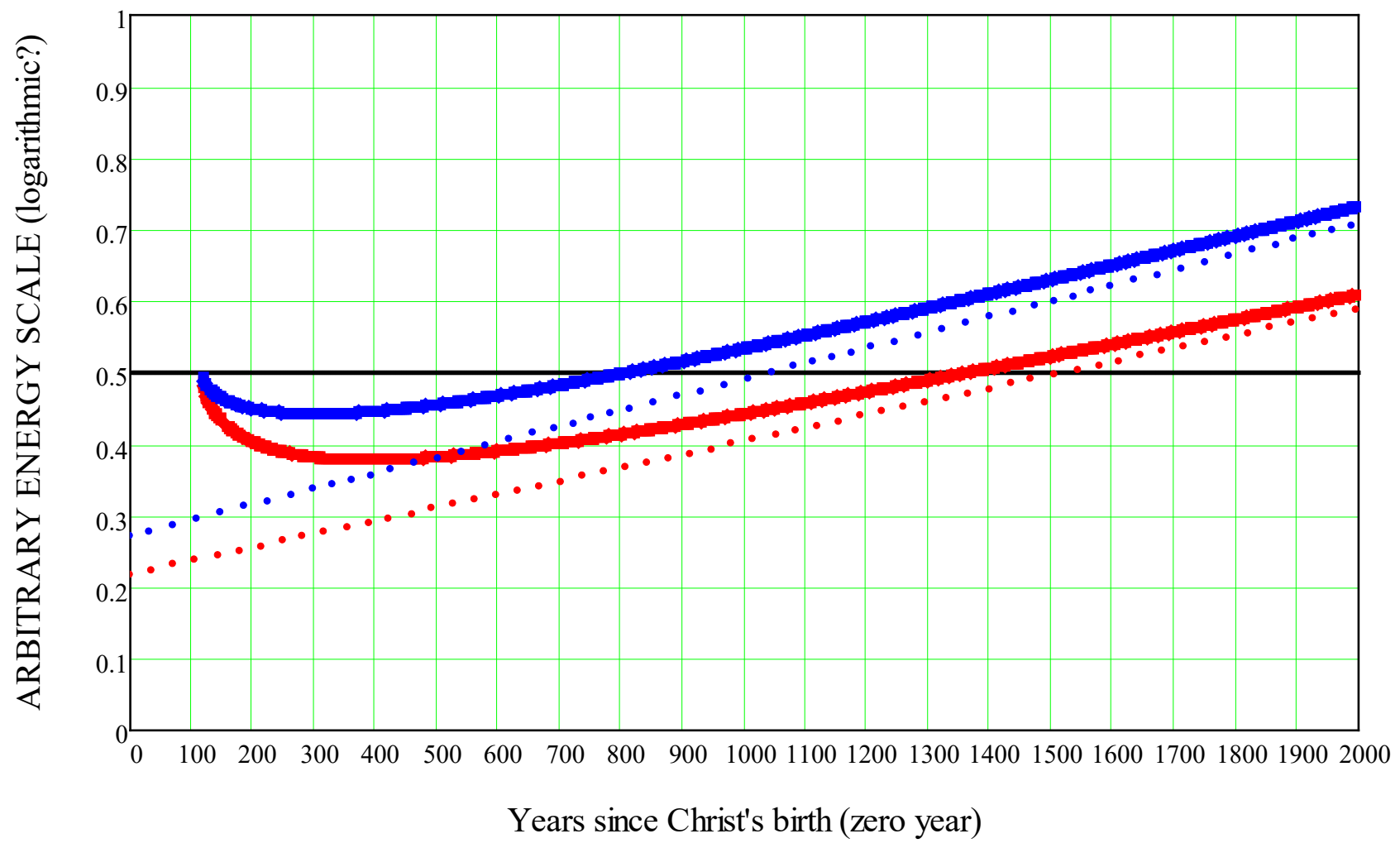

1) Figure 4. Rome's total energy as a function of $D$ (i.e., $d$, the death time regarded now as the independent variable): The solid curve in red is the LOGPAR ENERGY curve as described in ref. [1]. The dot-dot straight line in red is its oblique asymptote.

2) The solid curve in blue is the LOGELL ENERGY curve as given by equation (48) of this paper. The dot-dot straight line in blue is its oblique asymptote.

Now consider the definition of oblique asymptote given in elementary calculus textbooks: if the limit

$$
\lim _{D \rightarrow \infty}[\operatorname{Energy}(D)-(m D+q)]
$$

exists, then the Energy curve Energy $(D)$ approaches more and more the straight line

$$
y_{\text {oblique_asymptote }}(D)=m D+q . \quad \text { (50) }
$$

Differentiating (50) with respect to $D$, we immediately see that the angular coefficient $m$ of the oblique asymptote is given by the limit for $D \rightarrow \infty$ of the first derivative of the energy (48).

In fact, the first derivative of (48) is given by the following lengthy expression provided by Maxima:

$$
\begin{aligned}
& \frac{\mathrm{d}}{\mathrm{d} D} \text { logell_energy }=\left(\left(\pi^{2} D^{2}-4 \pi p D-2 \pi^{2} b D+4 \pi b D+\pi^{2} p^{2}-8 \pi p^{2}+16 p^{2}-2 \pi^{2} b p+20 \pi b p-32 b p+2\right.\right. \\
& \left.\left.\pi^{2} b^{2}-12 \pi b^{2}+16 b^{2}\right) \% \mathrm{e}^{2 \pi D+2 \pi p-8 p-4 \pi b+8 b}-\frac{\pi D}{2 \pi D+2 \pi p-8 p-4 \pi b+8 b}\right) /\left(2^{5 / 2}(p-b) \sqrt{D-p}\right. \\
& \left.(\pi D+(\pi-4) p+(4-2 \pi) b)^{3 / 2}\right)
\end{aligned}
$$


Taking the limit of $\left({ }_{51}\right)$ for $D \rightarrow \infty$, we get the energy asymptote's angular coefficient $m$ :

$$
m=\lim _{D \rightarrow \infty} \frac{d \operatorname{Energy}(D)}{d D}=\frac{\sqrt{\pi}}{2^{\frac{5}{2}} \sqrt{e}(p-b)}=\frac{0.1900}{p-b} . \text { (52) }
$$

The same would of course been found had we considered the limit

$$
\lim _{D \rightarrow \infty} \frac{\operatorname{Energy}(D)}{D}=\lim _{D \rightarrow \infty} \frac{m D+q}{D}=m .
$$

As for the asymptote's intercept with the vertical axis, $q$, (50) shows that it is given by the limit

$$
q=\lim _{D \rightarrow \infty}[\operatorname{Energy}(D)-m D] .
$$

Thus, (50), (52) and Maxima yielded the result

$$
q=\frac{1-e r f\left(\frac{1}{\sqrt{2}}\right)}{2}-\frac{\sqrt{e} \sqrt{\pi} p}{2^{\frac{5}{2}} \sqrt{e}(p-b)}+\frac{\sqrt{2} \pi-2^{\frac{3}{2}}}{4 \sqrt{e} \sqrt{\pi}} .
$$

In conclusion, the oblique asymptote to the Logell Energy (48) is given by

$$
y(D)=\frac{\pi D+\pi(p-2 b)-4(p-b)}{2^{\frac{5}{2}} \sqrt{e} \sqrt{\pi}(p-b)}+\frac{1-\operatorname{erf}\left(\frac{1}{\sqrt{2}}\right)}{2} . \text { (56) }
$$

\section{Part 3: Mean Power in a Logell Lifetime}

\section{Mean Power in a Logell Lifetime}

In this section we are going to consider the notion of mean value of a logell power curve. Having abandoned the normalization condition for our logell curves, clearly we may not use the same mean value definition of a random variable typical of probability theory. However, using the Mean Value Theorem for Integrals instead is easy. This is a variation of the mean value theorem, which guarantees that a continuous function has at least one point where the function equals the average value of the function.

To translate the Mean Value Theorem for Integrals into a mathematical equation holding for logell curves, we have to start from the Area equation, which is the logell energy equation (48) with $d$ replaced by $D$, and divide that area by the length of the $(D-b)$ segment in order to get the point along the vertical axis such that the area of the rectangle equals the Area. This is the required Mean Power Value over a lifetime and is given by 
Mean_POWER_over_a_lifetime $=\frac{A(b, p, D)}{D-b}=$

$$
\begin{aligned}
& =\frac{\text { logell total_energy }(D)}{D-b}= \\
& =\frac{1}{D-b} \cdot\left[\begin{array}{l}
\frac{\sqrt{\pi} \sqrt{D-p}}{1-\operatorname{erf}\left(\frac{\sqrt{2} \sqrt{\pi(D-b)+(\pi-4)(p-b)}}{\sqrt{D-p}} \sqrt{\pi(D-b)+(\pi-4)(p-b)} e^{-\frac{\pi(D-p)}{2 \pi D+(2 \pi-8) p+(8-4 \pi) b}}\right.} \\
+\frac{2^{\frac{5}{2}}(p-b)}{2}
\end{array}\right] .
\end{aligned}
$$

Just to check the correctness of (57), consider the limit of the Mean POWER over a lifetime (57) for $D \rightarrow \infty$. The calculation implies the use of L'Hospital's rule, and the result is

$$
\begin{aligned}
& \text { Asymptotic_Mean_Power_over_a_lifetime }= \\
& =\lim _{D \rightarrow \infty}(\text { Mean_Power_over_a_lifetime })= \\
& =\lim _{D \rightarrow \infty}\left(\frac{\text { logell_total_energy }(D)}{D-b}\right)= \\
& =\frac{\sqrt{\pi}}{2^{\frac{5}{2}} \sqrt{e}(p-b)} .
\end{aligned}
$$

But, this is, of course, the same as angular coefficient (52) of the logell energy asymptote since this angular coefficient is just the limit of the derivative of the logell total energy for $D \rightarrow \infty$; so, everything makes sense.

By this we have completed the study of the mean along the vertical axis, i.e., the power axis. However, one might still wish to find, in some sense, "the mean value of what lies on the horizontal axis," i.e., the lifetime mean value. That is done in the next section.

\section{Part 4: Logell Lifetime Mean Value}

\section{Lifetime Mean Value}

It is natural to seek for some mathematical expression yielding the mean value of a lifetime, meaning the mean value along the time axis of the $(D-b)$ time segment representing the lifetime of a living organism, or a civilization or even an ET civilization. We propose the following definition of such a lifetime mean value:

lifetime_mean_value $=$

$$
=\int_{b}^{p} t \cdot b_{-} \operatorname{lognormal}(t ; \mu, \sigma, b) d t+\int_{p}^{D} t \cdot \operatorname{ellipse}(t) d t=\text { (59) }
$$

inserting the b-lognormal (7) and the ellipse (5) into (59), the latter is turned into

$$
=\int_{b}^{p} t \cdot \frac{e^{-\frac{(\log (t-b)-\mu)^{2}}{2 \sigma^{2}}}}{\sqrt{2 \pi} \sigma(t-b)} d t+\int_{p}^{D} t \cdot P \sqrt{1-\frac{(t-p)^{2}}{(D-p)^{2}}} d t \text {. (60) }
$$

The first integral may be computed in terms of the error function $\operatorname{erf}(x)$ given by (13), and the result is 


$$
\begin{aligned}
\int_{b}^{p} t & \cdot \frac{e^{-\frac{(\log (t-b)-\mu)^{2}}{2 \sigma^{2}}}}{\sqrt{2 \pi} \sigma(t-b)} d t= \\
= & \frac{e^{\frac{\sigma^{2}}{2}+\mu}\left[1-\operatorname{erf}\left(\frac{\sigma^{2}-\log (p-b)+\mu}{\sqrt{2} \sigma}\right)\right]}{2}+ \\
& +\frac{b\left[1-\operatorname{erf}\left(\frac{\log (p-b)-\mu}{\sqrt{2} \sigma}\right)\right]}{2}=
\end{aligned}
$$

which may be further simplified by resorting to (8), with the result

$$
\begin{aligned}
& \int_{b}^{p} t \cdot \frac{e^{-\frac{(\log (t-b)-\mu)^{2}}{2 \sigma^{2}}}}{\sqrt{2 \pi} \sigma(t-b)} d t= \\
& \quad=\frac{e^{\frac{\sigma^{2}}{2}+\mu}[1-\operatorname{erf}(\sqrt{2} \sigma)]}{2}+\frac{b\left[1-\operatorname{erf}\left(\frac{\sigma}{\sqrt{2}}\right)\right]}{2} .
\end{aligned}
$$

Re-expressing now (62) in terms of the Logell History Formulae (35), it finally takes the lengthy but exact form given by Maxima as follows:

$$
\begin{gathered}
\int_{b}^{p} t \cdot \frac{e^{-\frac{(\log (t-b)-\mu)^{2}}{2 \sigma^{2}}}}{\sqrt{2 \pi} \sigma(t-b)} d t=\quad(63) \\
-\frac{\frac{3 \pi(d-p)}{(p-b) \% \mathrm{e}^{2((\pi-4)(p-b)+\pi(d-b))}} \operatorname{erf}\left(\frac{\sqrt{2} \sqrt{\pi} \sqrt{d-p}}{\sqrt{(\pi-4)(p-b)+\pi(d-b)}}\right)}{2}-\frac{\sqrt{\pi} \sqrt{d-p}}{3 \pi(d-p)} \operatorname{erf}\left(\frac{\sqrt{2} \sqrt{(\pi-4)(p-b)+\pi(d-b)}}{2}\right) \\
\frac{(b-p) \% \mathrm{e}^{2((\pi-4)(p-b)+\pi(d-b))}}{2}+\frac{b}{2}
\end{gathered}
$$

As for the second integral in (6o), i.e., the ellipse integral, it is promptly computed as follows:

$$
\int_{p}^{D} t \cdot P \cdot \sqrt{1-\frac{(t-p)^{2}}{(D-p)^{2}}} d t=
$$




$$
\begin{aligned}
& \left(\left(3 p^{3}-6 d p^{2}+3 d^{2} p\right) \operatorname{asin}\left(\frac{D-p}{p-d}\right)+2 p^{3}-6 d p^{2}+6 d^{2} p-2 d^{3}\right) P+\sqrt{-D+2 p-d} \sqrt{D-d} \\
& \left.\left(2 D^{2}-p D-3 p^{2}+4 d p-2 d^{2}\right) P\right) /(6 p-6 d)
\end{aligned}
$$

Inserting for $P$ its expression (36), after some rearranging, we conclude that the ellipse integral is given by

$$
\begin{aligned}
& \int_{p}^{D} t \cdot P \cdot \sqrt{1-\frac{(t-p)^{2}}{(D-p)^{2}}} d t= \\
& \left(\left(\sqrt{\pi(D-b)+(\pi-4)(p-b)} \% \mathrm{e}^{2(\pi(D-b)+(\pi-4)(p-b))}+\frac{\pi(p-D)}{\pi D+\pi p-4 p-2 \pi b+4 b}\right.\right. \\
& \left(\left(3 p^{3}-6 d p^{2}+3 d^{2} p\right) \operatorname{asin}\left(\frac{D-p}{p-d}\right)+2 p^{3}-6 d p^{2}+6 d^{2} p-2 d^{3}\right) /(\sqrt{2} \pi(p-b) \sqrt{D-p})+(\sqrt{-D+2 p-d} \sqrt{D-d} \\
& \left.\sqrt{\pi(D-b)+(\pi-4)(p-b)}\left(2 D^{2}-p D-3 p^{2}+4 d p-2 d^{2}\right) \% \mathrm{e}^{2(\pi(D-b)+(\pi-4)(p-b))}+\frac{\pi(p-D)}{\pi D+\pi p-4 p-2 \pi b+4 b}\right) /(\sqrt{2} \pi \\
& (p-b) \sqrt{D-p})) /(6 p-6 d)
\end{aligned}
$$

(65).

In conclusion, the mean lifetime is found by summing (63) and (65) and reads

lifetime_mean_value =

(66)

$$
\begin{aligned}
& -\frac{(p-b) \% \mathrm{e}^{\frac{3 \pi(d-p)}{2((\pi-4)(p-b)+\pi(d-b))}} \operatorname{erf}\left(\frac{\sqrt{2} \sqrt{\pi} \sqrt{d-p}}{\sqrt{(\pi-4)(p-b)+\pi(d-b)}}\right)}{2}-\frac{b \operatorname{erf}\left(\frac{\sqrt{\pi} \sqrt{d-p}}{\sqrt{2} \sqrt{(\pi-4)(p-b)+\pi(d-b)}}\right)}{2}- \\
& \frac{(b-p) \% \mathrm{e}^{\frac{3 \pi(d-p)}{2((\pi-4)(p-b)+\pi(d-b))}}}{2}+\frac{b}{2} \\
& \left(\left(\sqrt{\pi(D-b)+(\pi-4)(p-b)} \% \mathrm{e}^{2(\pi(D-b)+(\pi-4)(p-b))}+\frac{\pi(p-D)}{\pi D+\pi p-4 p-2 \pi b+4 b}\right.\right. \\
& \left(\left(3 p^{3}-6 d p^{2}+3 d^{2} p\right) \operatorname{asin}\left(\frac{D-p}{p-d}\right)+2 p^{3}-6 d p^{2}+6 d^{2} p-2 d^{3}\right) /(\sqrt{2} \pi(p-b) \sqrt{D-p})+(\sqrt{-D+2 p-d} \cdot \sqrt{D-d} \\
& \left.\sqrt{\pi(D-b)+(\pi-4)(p-b)}\left(2 D^{2}-p D-3 p^{2}+4 d p-2 d^{2}\right) \% \mathrm{e}^{2(\pi(D-b)+(\pi-4)(p-b))}+\frac{\pi(p-D)}{\pi D+\pi p-4 p-2 \pi b+4 b}\right) /(\sqrt{2} \pi \\
& (p-b) \sqrt{D-p})) /(6 p-6 d)
\end{aligned}
$$


Just to give a numerical example, let us find the mean lifetime of the Civilization of Rome. The first integral (63), by virtue of the Rome input triplet (37), yields the numeric value of the mean b-lognormal, i.e.,

mean_value_of_Rome_b-lognormal $=-35.6 \quad(67)$.

This means four years before the battle of Actium fought on 2 September 31 B.C.: a crucial event that saw Cleopatra's Egypt being absorbed as just one more Roman province ("Battle of Actium").

On the other hand, the quarter-of-ellipse integral (16) and the Rome input triplet (37) yield for the quarterof-ellipse mean value the year is

Rome_quarter-of-ellipse_mean_value $=49.1 . \quad(68)$

This year, 49 A.D., was a year falling during the empire of Claudius (41-54 A.D.) and, most importantly, was just about sixteen years after Jesus Christ had been crucified in Jerusalem. So, by summing up the two equations (67) and (68), we reach the important conclusion that the mean value of the overall Rome's LOGELL History power curve falls just around the year 13.5 A.D., i.e., within the time of Augustus, first Roman emperor (who died August 19,14 A.D.):

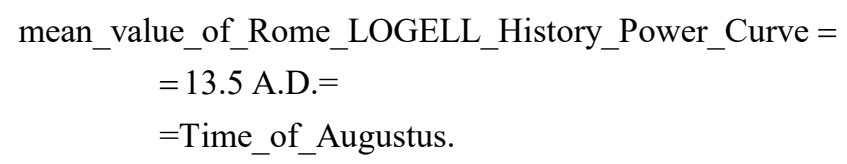

This is a noteworthy result. Our Evo-SETI Theory, in the LOGELL form described in this paper, predicts that the "most important year in the History of Rome happened ... just in the time of Augustus, and just when Jesus Christ was living his transition from a boy into an adult man"-By far the most important formation time in every man's life, and the more so in Jesus's own life!

Part 5: Conclusions: Which One Is Better? Logell or Logpar?

\section{Conclusions about Rome's Civilization}

In this paper we have discussed for the first time two alternative mathematical models for our Evo-SETI Theory:

1) The LOGELL model, where one's life is described by a power curve made up by a b-lognormal in between birth and peak plus a quarter of an ellipse between peak and death.

2) The LOGPAR model, where one's life is described by a power curve made up by a b-lognormal in between birth and peak plus descending parabola between peak and death.

Which one better describes the true history of a civilization? As for the case of Rome, the two models provide the following results:

1) LOGELL: minimum energy at the time of Diocletian, i.e., at the crucial transition between pagan and Christian Rome (313 A.D. Edict of Milan by Constantine and Licinius). Then, full recovery (at the old Trajan level of 117 A.D.) around the year 800 A.D. by Charlemagne.

2) LOGPAR: minimum energy around the year 378 A.D. (first serious defeat inflicted by the Barbarians to the Romans at the battle of Hadrianople) and full recovery only at the end of the Middle Ages about 1400 A.D. (Italian Renaissance).

The personal opinion of this author is that the LOGPAR model is more appropriate than the LOGELL one, at least in the case of Rome. 


\section{Conclusions about Evo-SETI Theory}

More and more exoplanets are now being discovered by astronomers either by observations from the ground or by virtue of space missions, like Kepler, Gaia, and other future space missions. As a consequence, a recent estimate sets at 40 billion the number of Earth-sized planets orbiting in the habitable zones of sunlike stars and red dwarf stars within the Milky Way galaxy. With such huge numbers of "possible Earths" in sight, astrobiology and SETI are becoming research fields more and more attractive to a number of young scientists. Mathematically innovative papers like the Evo-SETI ones, revealing unsuspected relationships like the one between the Molecular Clock and the Entropy of $b$-lognormals in Evo-SETI Theory, should thus be welcome.

In refs. [1], [2] and this paper we did more than just in all previous Evo-SETI papers. While just preserving all the advantages of the b-lognormal probability density functions, we kept these b-lognormals good for only the first part of the curve: the one between birth and peak. The second part, between peak and death, was replaced in the present paper for the first time by just a simple descending quarter-of-ellipse, thus avoiding any inflexion point like the "senility" point typical of b-lognormals that was so difficult to estimate numerically in most cases. Thus LOGELL curves, just as LOGPAR curves, have greatly simplified the description of any finite phenomenon in time like the lifetime of a cell, or a human, or a civilization (like the Rome one used in this paper as an example) or even like an ET civilization.

In other words, we abandoned the normalization condition of b-lognormals retaining just their shape but not the unit area underneath. This transformed both logpars and logells into power curves, both in the popular sense where "power" means political and military power and in the strictly physical sense, where "power" means a curve measured in Watts. The area under such a logpar or logell is indeed the ENERGY associated to the whole lifetime between birth and death. So, for the first time in the creation of our EvoSETI Theory, we were able to add ENERGY to the ENTROPY previously considered already. Energy and entropy are the two pillars of classical thermodynamics, making Evo-SETI even more neatly applicable to a host of biological as well as physical phenomena.

Finally, one more crucial step lies ahead, which we made by introducing both logpars and logells. Without mentioning it much so far, we actually stumbled into the PRINCIPLE OF LEAST ACTION. This is, of course, the \#1 mathematical tool of all theoretical physicists: just think of all the unified theories of gravitation, where a certain action function is postulated, then the Least Action Principle (or Hamilton's Principle) and the relevant Euler-Lagrange differential equations are derived, and finally (hopefully) solved, yielding the trajectory of particles. Well, the ACTION has the dimension of an ENERGY MULTIPLED BY THE TIME, and this is precisely what we did when finding the area under the logpars and the logells and considering their integrals between birth and death. Therefore, we claim that the logpars and logells are the optimal trajectory of our Evo-SETI Theory, also in regard to the Least Action Principle. The future will reveal whether our conjectures are right and largely applicable to astrobiology.

\section{References}

"Battle of Actium." 2020. Wikipedia: The Free Encyclopedia. Accessed March 2020.

https://en.wikipedia.org/wiki/Battle_of_Actium.

Maccone, C. 2018. "Energy of Extraterrestrial Civilizations according to Evo-SETI Theory." Acta Astronautica 144, 202-213.

-_- 2018. "Life Expectancy and Life Energy according to Evo-SETI Theory." International Journal of Astrobiology 18(1) 1-11. 